\title{
Particle flow simulation of sand under biaxial test
}

\author{
Xiao-li Dong ${ }^{1,2, a^{*}}$, Wei-hua Zhang ${ }^{1, a}$ \\ ${ }^{1}$ Beijing City University, China , ${ }^{2}$ Beijing Jiaotong University \\ adxlwu@163.com
}

\begin{abstract}
Keywords: sand, PFC2D model, a linear contact model
Abstract. Sand, also known as non cohesive soil, the particle is contact point to point. The particle properties are very suitable for the simulation of particle aggregation through the biaxial tests. Study on the sand mechanical parameters by the biaxial test with a linear contact model, parameters of the study include: confining pressure, particle friction coefficient, the particle stiffness, particle size ratio. And the comparing with different parameter curve of peak strength, strain and secant modulus.
\end{abstract}

\section{Introduction}

The conventional particle flow model can be used to simulate the mechanical behavior of a system composed of the arbitrary shaped particles that are independent of each other. If the particle is considered as a rigid body, the contact surface is treated as a soft contact surface to achieve the effect of normal finite rigidity. The mechanical behavior of the system can be described by the displacement of each particle and the interaction force between the particles at each contact point. Newton's law of motion establishes the relationship between the particle motion and the force of motion. The system may be static equilibrium (in this case, the system does not move) or makes the particles flow.

A PFC2D model is composed of a set of 2D circular particles. The particles described by PFC2D are only two dimensional, i.e., only two forces and one moment, which is different from the three-dimensional particles. The force outside the plane and the two moments in the plane are not considered in any case in the equations of motion or Hooke's law.

The basic unit movement in the PFC2D program follows the second Newton's law and the contact between the basic units abide by the law of force and displacement.. The calculation of the program is to divide the time step by step. Contact between particles or particles and the "wall" abide by the law of force and displacement.

The constitutive model of the contact point of the particles can be divided into three kinds: the contact stiffness model, the contact slip model and the contact bond model. Elastic relationship between the contact force and relative displacement in the stiffness model. The slip model provides the relationship between normal force and tangential contact force. The cementation model considers the discrete particle element to be bonded together, which limits the total normal force and tangential force. The application of the cement model is extended to solve the mechanical problems of continuous medium and continuous medium to discontinuous medium, such as damage of concrete, crack propagation of rock and soil, and so on.

PFC2D provides two kinds of contact stiffness models: linear model and simplified Hertz-Mindlin model. This paper focuses on the linear model.

\section{Study on the sand mechanical parameters by the biaxial test with a linear contact model}

As the particle flow sample is actually based on the microscopic characteristics of granular media, the interaction between particles in the PFC2D numerical model reflects the micro mechanical behavior of the granular media. The macro view of the soil is based on the simulation of granular flow, and the following simulation uses linear contact model.

In this paper, the influence factors of mechanical properties of sand by using the method of PFC2D.

The particle parameters include the particle density $\rho_{g g}$, the average particle size of $\mathrm{R}$, the maximum 
and minimum particle diameter ratio, porosity $n$, the particle friction coefficient, the tangent stiffness $k_{n}$ and the shear stiffness $k_{s}$

Sand sample preparation: choose $0.0945 \mathrm{~m} \times 0.0377 \mathrm{~m}$, and the size is very close to the actual conventional three axis specimen size. The particle unit use the two-dimensional disk unit, the average particle size of $0.001 \mathrm{~m}$. The number of particle is 997 , the initial porosity is 0.12 , particle density is $2650 \mathrm{~kg} / \mathrm{m}^{3}$, uniform distributing and $\mathrm{rmax} / \mathrm{rmin}=4$. and they are.generated by the radius expansion method. The sample is loaded with strain control mode, and test loading rate is constant. The upper and lower wall is moving with $0.4 \mathrm{~m} / \mathrm{s}$ loading rate, and consolidation samples confining pressure $2 \mathrm{MPa}$ using isotropic consolidation, and the test uses drained shear conditions, numerical sample parameters are shown in Table 1 and Table 2.

Tab. 1 Parameters table of the numerical simulation sample

\begin{tabular}{c|c|c|c|c|c|c}
\hline $\begin{array}{l}\text { Sample } \\
\text { size/mm }\end{array}$ & $\begin{array}{l}\text { Particle } \\
\text { density/kg.m } \\
3\end{array}$ & $\begin{array}{l}\text { M aximum } \\
\text { size/mm }\end{array}$ & $\begin{array}{l}\text { M inmum } \\
\text { size/mm }\end{array}$ & $\begin{array}{l}\text { Initial } \\
\text { porosity }\end{array}$ & $\begin{array}{l}\text { Number of } \\
\text { particles }\end{array}$ & $\begin{array}{l}\text { confining } \\
\text { pressure } \\
\text { M pa }\end{array}$ \\
\hline $94.5 \times 37.3$ & 2650 & 0.16 & 0.04 & 0.12 & 997 & 2 \\
\hline \multicolumn{3}{c|}{ Tab.2 Mesoscopic parameters for sand samples } \\
\hline & $k_{n}(\mathrm{~Pa})$ & $k_{s}(\mathrm{~Pa})$ & $\mu$ \\
\hline & $4 \mathrm{e} 8$ & $4 \mathrm{e} 8$ & 0.5 \\
\hline
\end{tabular}

Parameters of the study include: confining pressure, particle friction coefficient, the particle stiffness, particle size ratio. And the comparing with different parameter curve of peak strength, strain and secant modulus.

\section{Consideration of confining pressure}

Under different confining pressures ,such as 4Mpa, 6Mpa, 8Mpa, 10Mpa, the relationship of shear stress, axial strain, volumetric strain and axial strain, structure and shear stress ratio; normal stress and phase void ratio are shown in Fig.1 to Fig.3 .

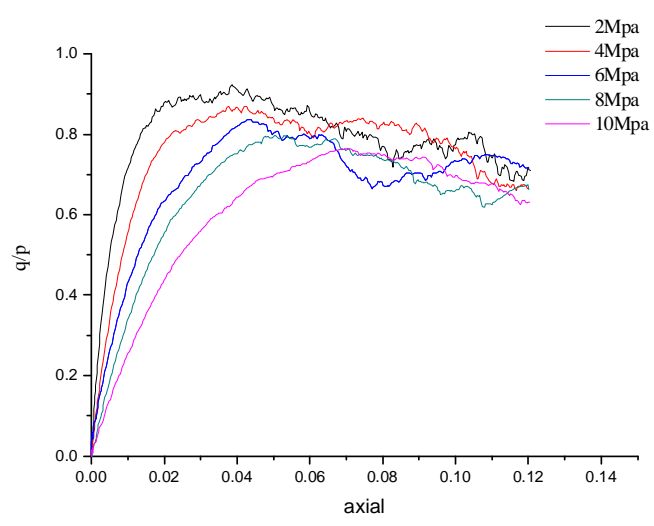

Fig. 1 Relationship between destress/stress ratio vs strain under different confinning pressure

It can be found that with the increase of the confining pressure, they all increase:

1) The peak strength and the corresponding strain of sample increased significantly, the deformation modulus increased slightly;

2) The peak stress ratio is smaller, the stress ratio and the axial strain curve are more and more slowly, and the strain softening stage is weakened;

3) The corresponding axial strain increases gradually, and the corresponding amount of compression increases with the increase of the amount of the phase transition. Then the shear expansion trend is the same. 
4) The same initial state samples under different confining pressure can get the phase change of the ratio of the normal stress in the semi-logarithmic coordinates with the corresponding positive stress.

5) The anisotropy coefficient of sample particle contact normal decreases slightly. Under the same confining pressure, the anisotropic coefficient of the contact normal force and the stress ratio of the particles are linear.
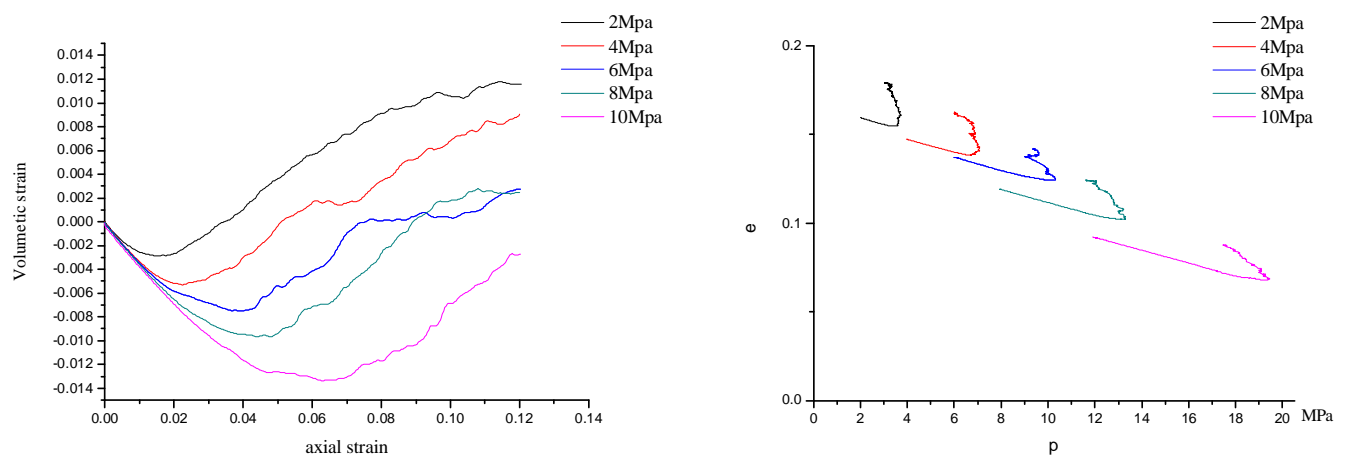

Fig.2 Relationship between volumetic strain, void ratio vs axial strain under different confinning pressure

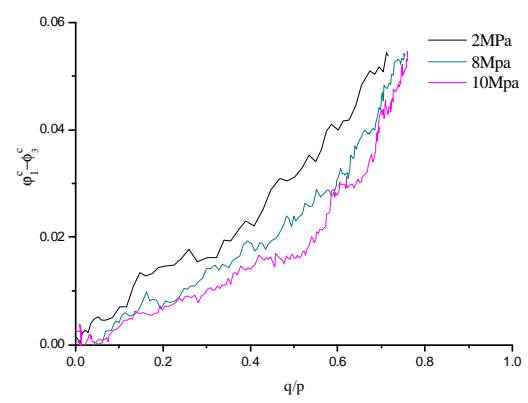

Fig.3 Relationship between phase trasformation void ratio and corresponding fabric parameter $\left(\varphi_{1}^{c}-\varphi_{3}^{c}\right)$ and deviatoric stress ratio under different confinning pressure for sands

\section{The effect of friction coefficient}

As shown in Fig 4, The peak values of the stress-strain curves can be improved obviously, and the initial elastic modulus increases with the increase of friction coefficient. The greater the friction coefficient, the more obvious the softening phenomenon. And the smaller friction coefficient corresponds to the ideal elastic-plastic relationship. From the relation of the volumetric strain to axial strain, the greater the friction coefficient, the greater the compression, and the shear expansion in the later became more obvious.
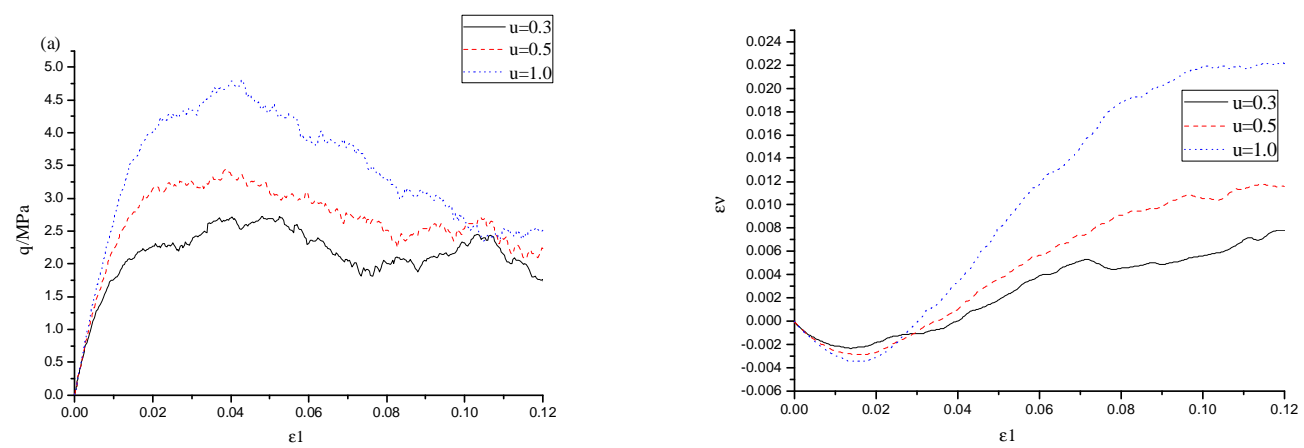

Fig.4 Effect of frictional parameters on the mechanical behavior of sands 


\section{The influence of particle contact stiffness on the stress-strain curve}
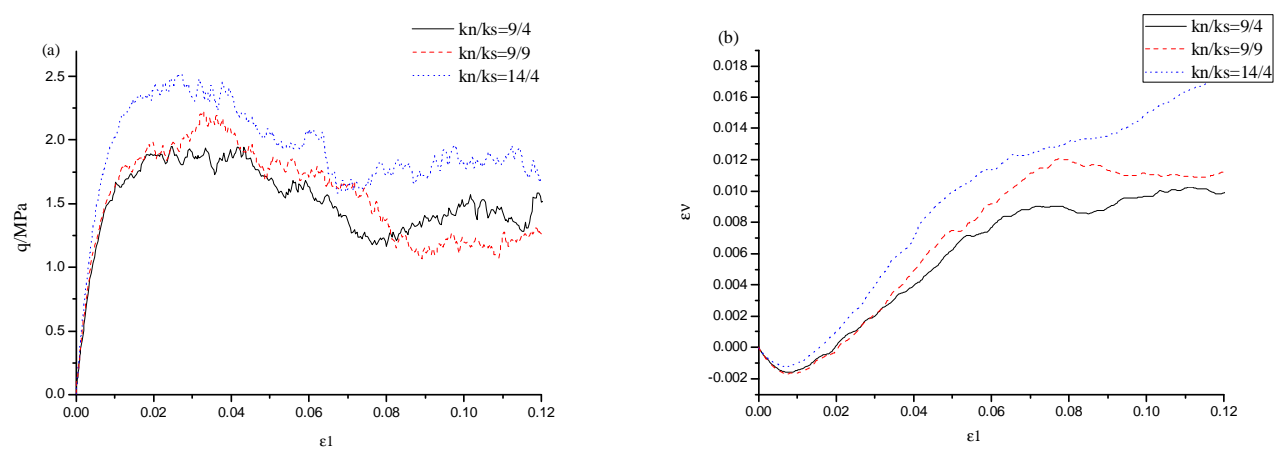

Fig. 5 Effect of thecontact stiffness between particleson the mechanical behaviour of sands

From Figure 5, it can be seen that $\mathrm{kn}$ and $\mathrm{k}_{\mathrm{S}}$ have a great influence on the stress-strain curve of sand. Obviously, the initial elastic modulus increases with the increase of normal contact stiffness, and the change of tangential contact stiffness ks has little effect on the initial elastic modulus, and the stiffness ratio directly affects the change of Poisson's ratio.
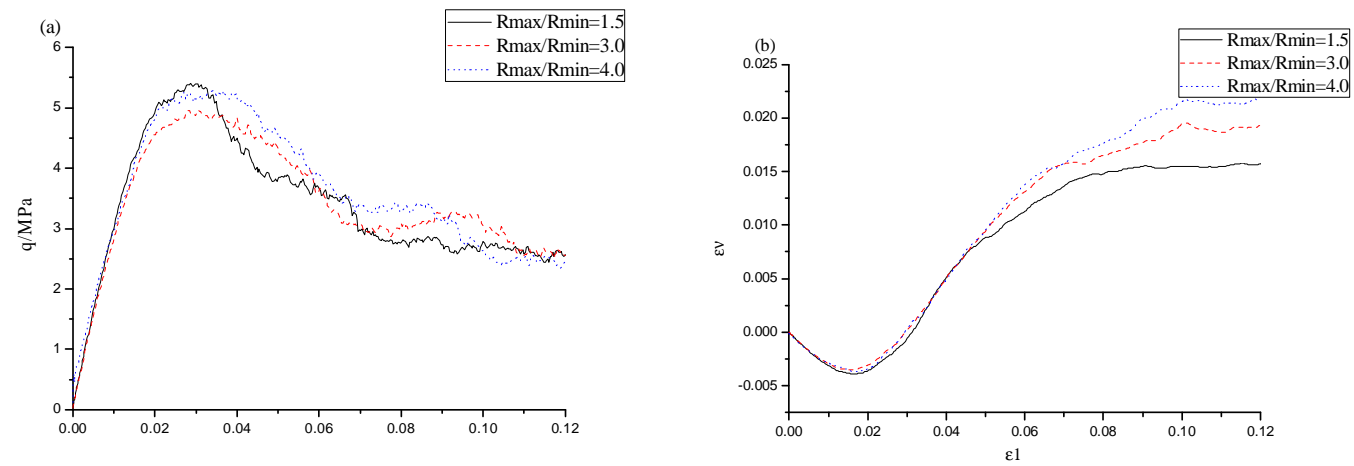

Fig. 6 Effect of ratio of Rmax/Rmin on the mechanical behavior of sands

\section{Effect of particle size on the mechanical behavior of sand}

Minimum particle size of the sample is not much affected by the stress strain curve of the sample. And the Fig6 (b) shows that the particle size ratio has no effect on the location of the phase transition of sand, but it has a great influence on the later period of shear.

\section{conclusions}

In the simulation of the three axis test results of standard sand, the linear contact model is used to obtain a satisfactory result for a certain confining pressure level. The peak strength, volume change, structure and phase change stress ratio of the "sand" are influenced by the confining pressure level, the particle friction coefficient, the stiffness ratio and the particle shape factor.

\section{References}

[1] Zhou jian,Chi yuwei,Chi yong,Xu jianping. Simulation of biaxial test on sand by particle flow code. Chinese journal of geotechnical engineering, 2000, 22(6). 701-704

[2]Bowmanet,Sogak,Drummondw.Particle shape characterisation using Fourier analysis[J]. Geotechnique , 2000 , 51(6) : 545-554. 
[3] Kong Liang, Peng Ren, Particle flow simulation of influence of particle shape on mechanical properties of quasi-sands [J]. Chinese Journal of Rock Mechanics and Engineering , 2011,30(10):2112-2119.

[4]Shi danda,Sand mechanical properties and microscopic simulation Under monotonic and cyclic loading[D]. master's degree thesis of Tongji University, 2007 